\title{
Automatic recording of various aspects of foraging behaviour
}

\author{
SM Rutter, RA Champion, PD Penning \\ Institute for Grassland and Environmental Research, North Wyke, Okehampton, Devon EX20 2SB UK
}

An understanding of plant resource use by grazing ruminants requires accurate, noninvasive measurements of foraging behaviour. An automatic, computer-based system has been developed to record various aspects of the foraging behaviour of free-ranging herbivores.

The animal behaviour recorder is based on a miniature, single-board microcomputer (Triangle Digitał Services Limited TDS2020). A conductive rubber nose-band senses the animal's jaw movements. The analogue signal from the nose-band is digitised by the microcomputer. Digital, 8 bit samples are recorded at a frequency of $20 \mathrm{~Hz}$ onto a $2 \mathrm{Mb}$ static ram card. Subsequent processing of these samples can be used to determine when the animal was grazing and when it was ruminating. Individual prehension bites and mastications during grazing and individual mastications during ruminating can be identified and counted. Mercury tilt switches suspended beneath the animal and attached to its leg can be used to detect whether the animal is lying or standing and when it is walking. These sensors are sampled at 0.5 and $20 \mathrm{~Hz}$ respectively, and the data are stored along with the jaw data on the $2 \mathrm{Mb}$ ram card. The recorder is powered by a rechargeable 7.2 v 1.7 Ah Nickel-Cadmium battery, and can record for a period of up to 25.5 hours. The recorder weighs $1.5 \mathrm{~kg}$ (including battery pack and harness) and the electronics are enclosed in a robust, waterproof (IP65) enclosure $(120 \times 120 \times 60 \mathrm{~mm})$.

Alternatively, the recorder can be configured to record 1 bit samples of jaw activity (i.e. either active, 1 , or inactive, 0 ) at $0.5 \mathrm{~Hz}$. These samples can be processed to determine when the animal was eating and when it was ruminating, but cannot be used to identify individual prehension bites or mastications. However, the low sampling rate allows the recorder to collect data for periods of up to eight days when powered by five nonrechargeable $1.5 \vee 2.7$ Ah alkaline batteries.

A Global Positioning System (GPS) satellite navigation receiver can be incorporated into the system to give the animal's location. The basic accuracy of the GPS is $100 \mathrm{~m}$, but this can be improved to $5 \mathrm{~m}$ (for more than $95 \%$ of the time) using a technique known as differential correction. The first version of the system used a Trimble Navigation Limited SVeeSix GPS receiver. This was operated on a $50 \%$ power cycle (i.e. on 30 seconds, off 30 seconds) and gave the animal's position (latitude, longitude and altitude) each minute. This was logged onto the $2 \mathrm{Mb}$ ram card along with jaw activity and lying/standing data. The system was powered by a non-rechargeable $7.0 \vee 39.0$ Ah Lithium battery, and could log data for a period of up to 7 days. Recent advances in GPS receiver technology now allow decimetre accuracy.

This animal behaviour recorder allows the accurate recording of various aspects of the foraging behaviour of free-ranging herbivores. Information about animal movements and whether they are lying or standing can be used to estimate energy expenditure. Information about patterns of grazing and animal position can be used to identify the areas grazed by herbivores. Although the system has, to date, only been used with domestic sheep and cattle, it can be used to record the foraging behaviour of any vertebrate above a minimum size. 